\title{
Unification Process for Uniforms with Use of 3D Body Scanning
}

\author{
Łukasz MARKIEWICZ*1 Elżbieta MIELICKA ${ }^{2}$, Gabriel WASILEWSKI ${ }^{3}$, \\ Krzysztof MULARCZYK ${ }^{1}$, Lidia NAPIERALSKA ${ }^{2}$, Robert SITNIK ${ }^{1}$ \\ ${ }^{1}$ Warsaw University of Technology, Mechatronics Faculty, Warsaw, Poland; \\ ${ }^{2}$ Textile Research Institute, Knitting and Clothing Research Department, Lodz, Poland; \\ ${ }^{3}$ Medcore Sp. z 0.0 [Ltd.], Warsaw, Poland \\ DOI: 10.15221/17.170 http://dx.doi.org/10.15221/17.170
}

\begin{abstract}
There is much to be done to improve fitting quality of uniforms used by uniformed services in Poland. The main reasons of badly fit uniforms are inconsistency of uniform sizes in each of the services and statistical differences in body measures since the last national population survey. In order to develop the new statistics of body sizes and size charts, 3D measurements of body shape have been carried out on a large scale. Additional goal was to develop the OGX|MMS measurement system which could be used by the services in the future for periodic monitoring.
\end{abstract}

The developed system's working principle is structured light projection. The scanning system is composed of four measurement heads, each containing two 3D scanners placed vertically. Due to a spectral separation of the scanners, all measurement heads can perform data acquisition at the same time. Total measurement time is approximately $3 \mathrm{~s}$. The result is a 3D cloud of points which represents the measured body surface with resolution of $1 \mathrm{~mm}$ and accuracy below $0.3 \mathrm{~mm}$.

New algorithms were also developed for extracting anthropometric measurements from acquired body models. During the project 34 types of measurements were carried out to enable the creation of new size charts. The processing path for the measurements included filtration and measurement noise elimination, 3D segmentation, location of anatomical landmarks and calculation of body measurements (arc lengths, girths and linear sizes). A commercial Human Solutions laser line-based 3D measurement device was used as a reference for the automatically extracted measures. Comparative analysis between the two 3D scanning methods and manual measurements was performed. Manual measurements were taken according to the ISO 7501-1, ISO 8559, and EN 13402 standards. 26 features which can be used for clothing design were chosen for the analysis.

The efficiency of the scanning process was tested in a real-life environment. The main advantage of the 3D scanning method compared to the manual measurements is the ease and speed of the process and the ability to repeat measurements of the specific features using the stored virtual model.

Keywords: 3d body scanning, anthropometry, size designation, measurements, automated analysis

\section{Introduction}

One of the numerous groups affected by the outdated size designation systems are Polish officers belonging to the Police, Border Guard, Government Protection Bureau, and Fire Service. The last sizing charts update for officer formations in Poland was done in mid 80s. Another drawback of the currently used standards is that each officer formation has its own system for size designation which does not correspond to actual officers' body dimensions. At the same time, the number of women in these formations grew, so there is an additional need to prepare a gender-based sizing system. In order to solve this problem, the Polish National Centre for Research and Development (NCBIR) launched a project named "Formfit—Anthropometric studies of officers employed by the Polish Ministry of the Interior (MSW)".

The aim of this article is to present an approach to unification of uniforms based on the Formfit project case, along with tools, systems and methods incorporated into this research. As every unification process for clothing, also this had to be preceded by population-wide anthropometric surveys. Maintaining an up-to-date anthropometric database of a representative population is crucial for the correct garment fitting. Traditional manual anthropometric approach is a very tedious, expensive, and long-lasting task. Therefore it was decided to utilize modern 3D anthropometry techniques, as it could speed-up and facilitate this process at lower costs. The unification process was divided into 5 main

*I.markiewicz@mchtr.pw.edu.pl; (+48) 664602550 
parts, i.e. interprofessional consultations on the requirements and methodology; choice of the commercial 3D anthropometric reference system and development of the new, fully customized one; preliminary validation of measurement algorithms; practical tests where officers were scanned and for whom tailored clothing is being prepared; final statistical analysis resulting in updated statistics of body sizes and size charts. The most challenging part of the work during this project was to develop a reliable, comprehensive 3D anthropometric solution from scratch. It consisted of a 3D mobile whole body scanner equipped with an automatic measurement extraction and analysis software. The scanning results were then linked with the statistical analysis platform using a remote database. The newly developed 3D anthropometric scanner was based on the structured light projection technique $[1,2]$. It was composed of four measurement heads, each containing an upper and a lower 3D scanner, placed vertically. For the purpose of this research, new anthropometric measurement algorithms had to be developed and tested. Their preliminary validation was performed on a group of 40 volunteers (21 male and 19 female). They were manually measured by a trained operator and scanned three times each by both reference, commercial Human Solutions system and the newly developed one. Quality assessment of the results proved that the proposed system's performance is comparable to that of the state-of-the-art solution and can be used for further research.

The next stage of the project included real-life environment validation of the results. During the practical tests, 32 real officers were scanned and are expecting to receive tailored uniforms. Simultaneously, Medcore, an IT company which is a consortium member in the project, developed the database and statistical software. Afterwards, the exact population scanning survey was conducted using both available 3D anthropometric solutions. Altogether, 3364 officers from four different formations were fully scanned, in which 530 scans were performed by the newly developed system, and the rest by the Vitus 3D body scanner from the Human Solutions system. The final, still ongoing stage, focused on new statistics of body sizes and corresponding size charts. Despite that it is not finished yet, early results proved the validity and advisability of the project. Preliminary statistical analysis revealed that for some of the garment types real body dimensions of even every second officer does not fit into any of the existing size tables. This means financial losses and deterioration of comfort of wearing the garment. This research proves that it is possible and worth to utilize modern 3D scanning technologies for large-scale surveys.

\section{Materials and methods}

Despite being faster and more effective than the traditional approach, unification of uniforms with use of $3 \mathrm{D}$ whole body scanning is still a time-consuming, non-trivial, and multi-stage process because of its scale and complexity. It requires a combined effort of several groups of experts, in particular textile researchers and engineers responsible for the quality of the 3D-derived measurements. Furthermore, the clothing needs of the specific population have to be taken into account. The stages of the unification process conducted during this research are presented below (see figure 1).

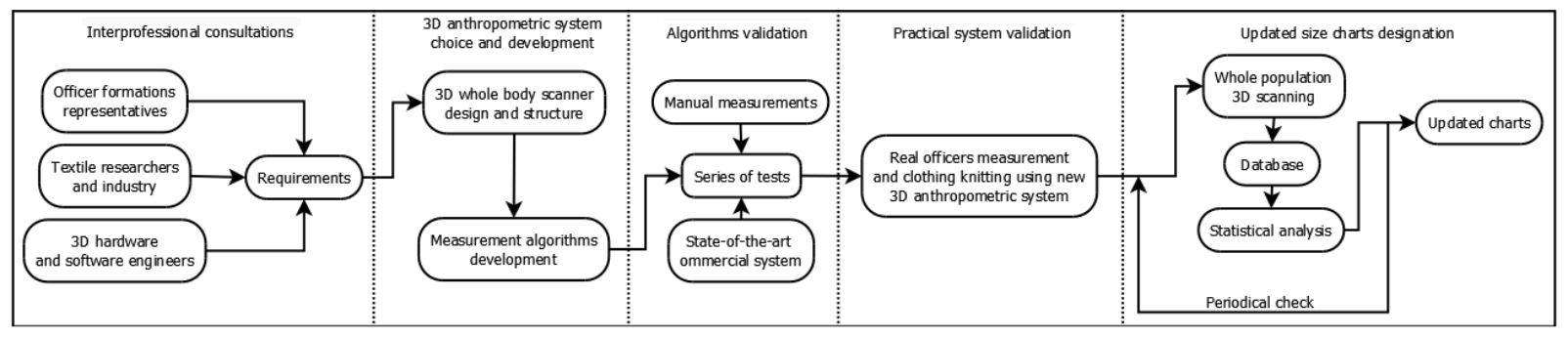

Fig. 1. Uniforms unification process stages diagram.

\subsection{Interprofessional consultations - defining requirements}

The first stage of the process began with the interprofessional consultations between experts on what to measure and how. In order to provide optimal updated garment size charts, the Textile Research Institute (Poland) selected 34 desired measurements (see figure 2). The choice was ensured to be compliant with the international standards, i.e. ISO 7501-1, ISO 8559, and EN 13402 as well as with the Polish recommendations for anthropometric measurements [3]. The other issues, e.g. scanning procedure, prerequisites, subject position during scanning were also resolved in order to minimize the number of erroneous scans. The scanned person had to be undressed to tight, bright undergarments and take the proper, straddle position with upper limbs apart from their torso. During the scanning, subject had to stay still for $3 \mathrm{~s}$ which was enough to capture all of the body surface. 
The next goal was to develop a 3D measurement system which could automatically obtain all of the measurements in an expected way and could be used by the services in the future for periodic monitoring. The new system, named OGX Mobile Measurement System (OGX|MMS), had to be built from the ground up. This ensured full customizability and process transparency in comparison to closed commercial solutions. A series of test measurements were carried out manually by trained operators and automatically by the OGX|MMS as well as the other, commercial state-of-the-art scanning system, to validate the developed device.

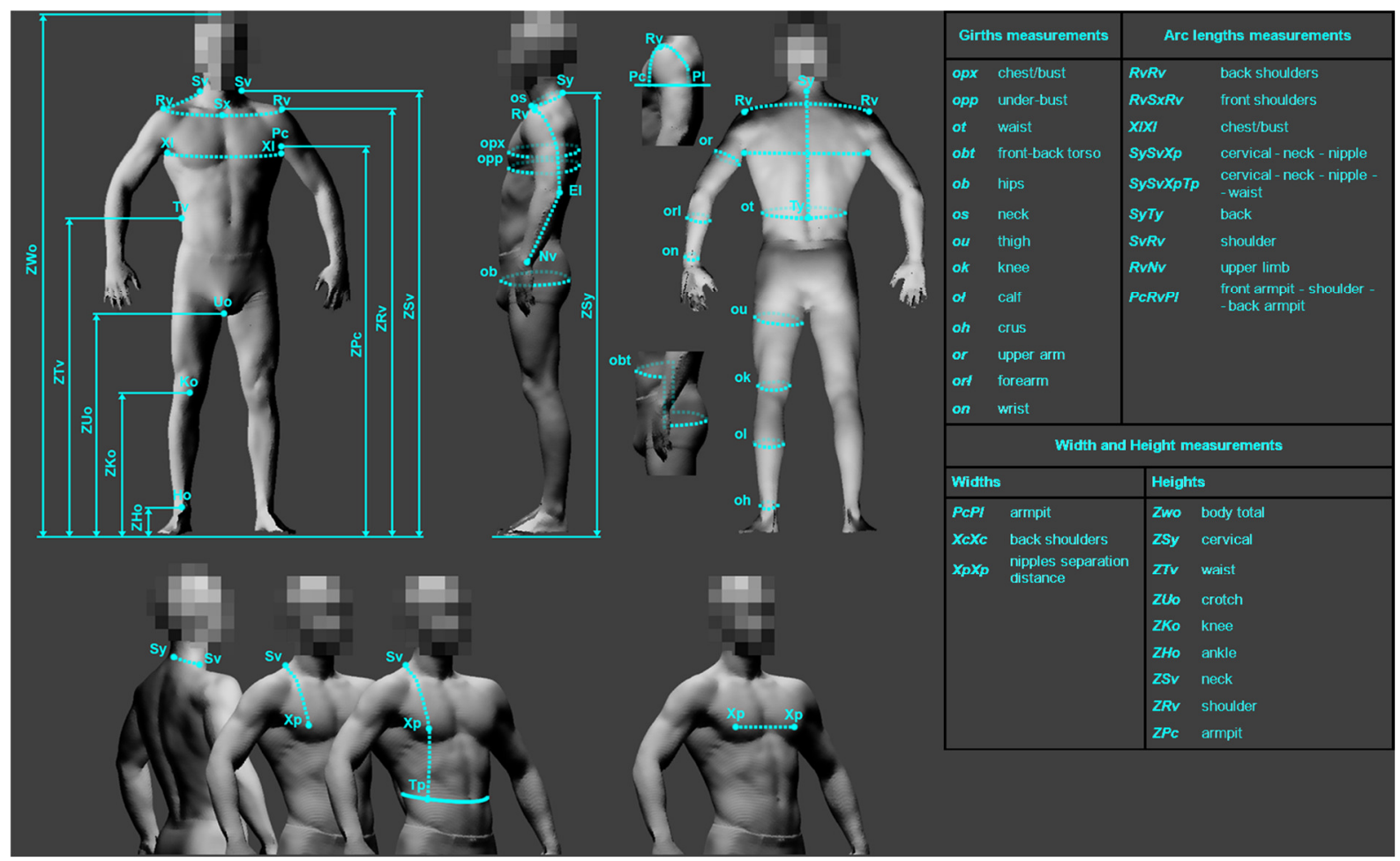

Fig. 2. Visualization of the 34 desired anthropometric measurements with its definitions.

\subsection{D anthropometric system choice and development}

The specialized 3D whole body scanner is a key part of every 3D anthropometric solution. Unfortunately, none of the systems available on the market provided complete desired functionality and fully addressed the project needs. Therefore, there was a need to develop a custom, comprehensive system which could fulfill all of the requirements.

\subsection{1. $3 D$ body scanner design and structure}

The OGX|MMS 3D whole body scanner working principle was based on a structured light projection. The system had to be easy to transport and install which is important, as the scanned formations were spread across the country. It was possible thanks to four measurements heads, each containing two detachable upper and lower parts, each equipped with a full-field 3D scanner. All of the modules were able to perform measurements simultaneously from opposite directions due to spectral separation of projector beams. This way, whole body surface could be captured in a short time (around $3 \mathrm{~s}$ ). The main components of each module were:

- two Charge Coupled Device (CCD) 1 MPix FireWire cameras (Point Grey Research Flea FL3-FW-14S3M-C);

- two Digital Light Processing (DLP) projectors (Casio XJ-A250);

- a network switch;

- a laser pointer; and

- a PC-class computer.

The projectors had to be customized to ensure proper repeatability and accuracy of measurements. In order to preserve stable parameters of the optical system, projector lenses were replaced by custom-designed ones. Moreover, additional reinforcement of the whole system had to be done to 
minimize the influence of vibrations caused by the cooling fans. The measurement heads were also equipped with laser pointers for use during calibration of the system and estimation of the scanning volume center during installation. The modules were connected with LAN cables and set up in the corners of a dimmed room. The OGX|MMS system was also equipped with a Mensor WM150P1 $40 X 50 G$ scale, additionally serving as a platform during scanning.

The OGX|MMS 3D scanner accuracy was evaluated using international norms. The maximum permission error was calculated according to ISO 10360-8 calibration norm and PTB VDI/VDE 2634 recommendations. A calibration object (ball bar - two spaced balls connected by a bar) was measured firstly by a coordinate measuring machine and then, in several positions, by our scanner. Afterwards, flatness was analyzed and final error was estimated.

Nevertheless, an additional, commercial state-of-the-art scanning system was needed for validation purposes, both during early OGX|MMS development stage and large-scale population scanning. A commercial Human Solutions laser line-based 3D anthropometric solution was chosen as a reliable reference [4]. The particular device was a Vitronic VITUS smart XXL 3D body laser scanner equipped with Anthroscan ScanWorX analysis software [5]. Comparative perspective views of both systems are shown in figure 3. Comparison between metrological specifications for both 3D whole body scanners is presented in table 1.
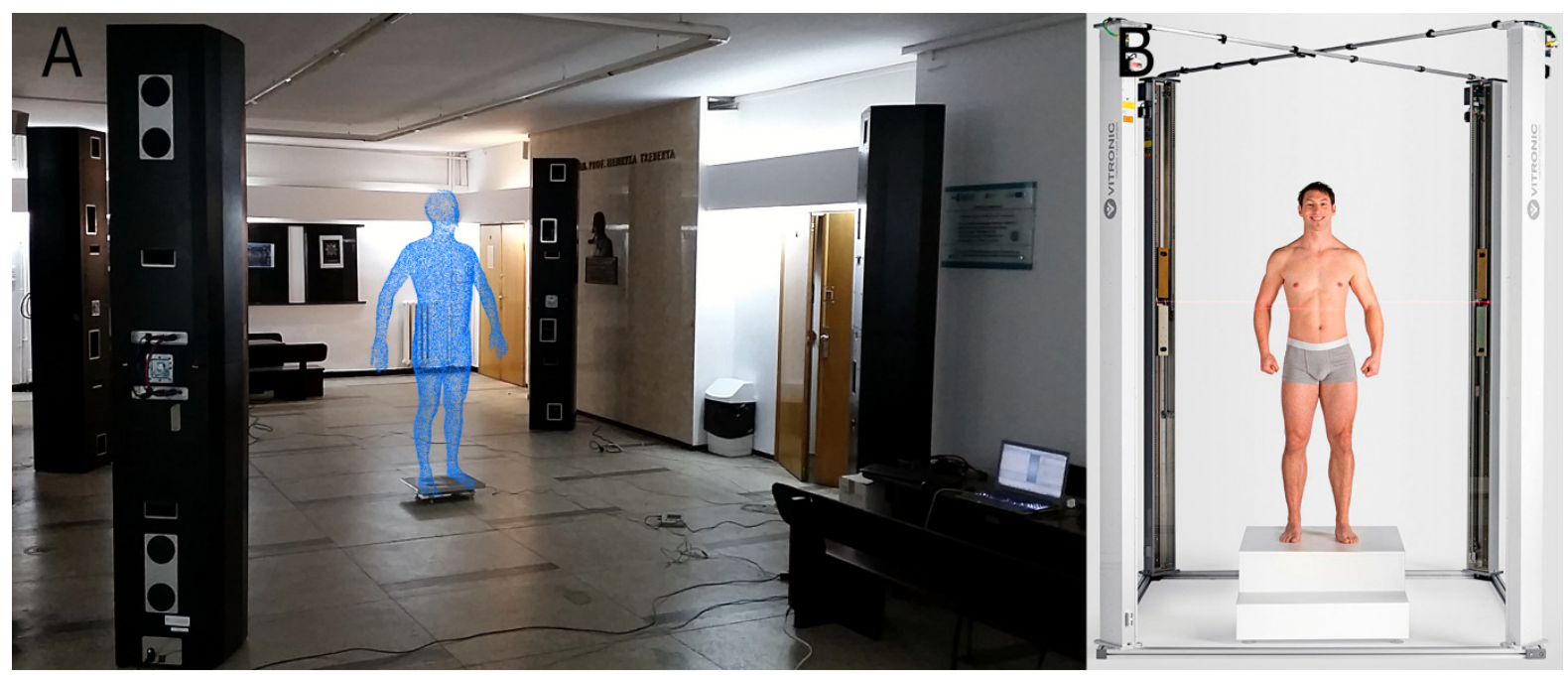

Fig. 3. Perspective views of OGXMobile Measurement System (A) and Vitronic VITUS smart XXL 3D body scanner from Human Solutions (B) [6].

Table 1. 3D whole body scanners metrological specifications.

\begin{tabular}{|l|l|l|}
\hline & OGX|MMS & VITUS smart XXL \\
\hline accuracy & less than $0.3 \mathrm{~mm}$ & less than $1 \mathrm{~mm}$ \\
\hline data acquisition time & around $3 \mathrm{~s}$ & around $10 \mathrm{~s}$ \\
\hline measurement volume size (LxWXH) & $1.5 \mathrm{~m} \times 1.5 \mathrm{~m} \times 2.2 \mathrm{~m}$ & $1.2 \mathrm{~m} \times 1.0 \mathrm{~m} \times 2.1 \mathrm{~m}$ \\
\hline number of points & from 1 to $2{ }^{*} 10^{6}$ & around $0.4^{*} 10^{6}$ \\
\hline
\end{tabular}

\subsubsection{Measurement algorithms development}

In order to automatically retrieve the desired measurements from acquired body models, numerous methods were implemented. Figure 4 presents the proposed generalized processing path. 


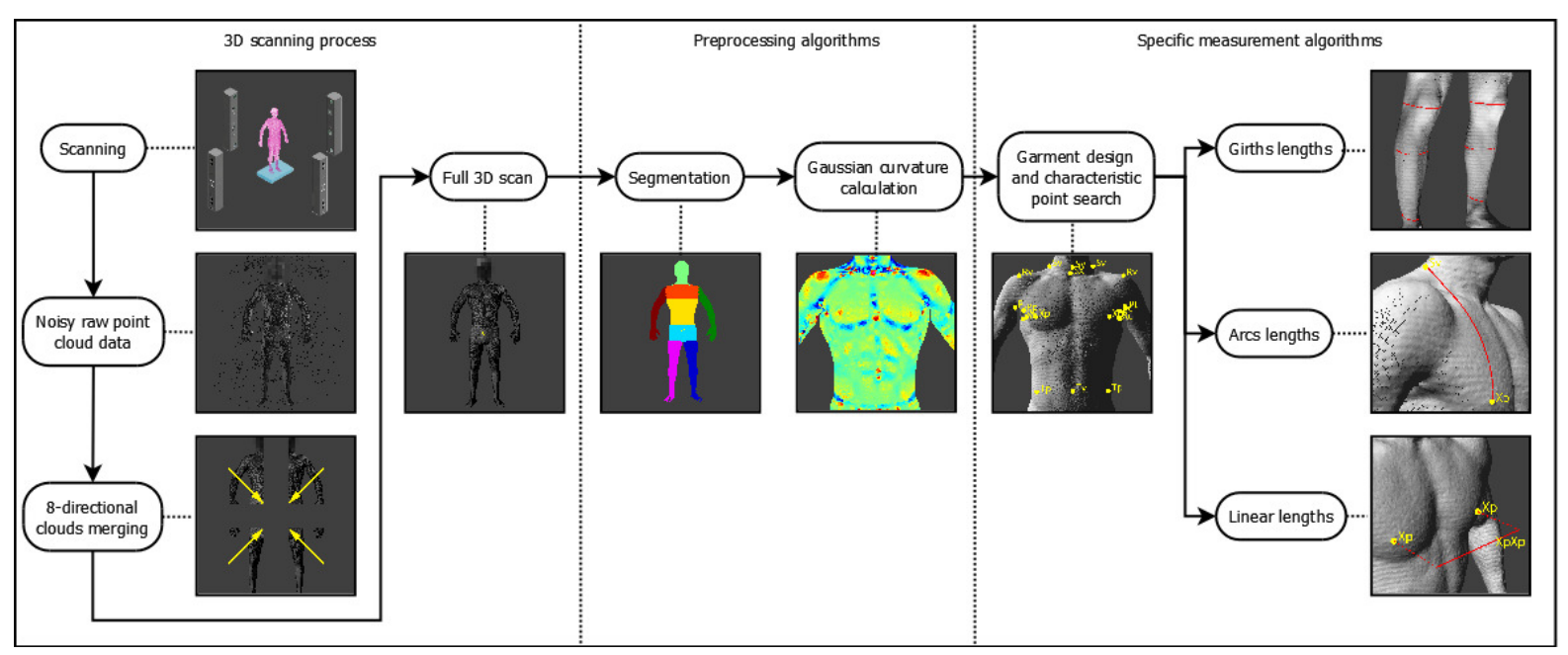

Fig. 4. General processing path diagram.

Raw output from the OGX|MMS scanner heads consisted of a set of 8 point clouds. First, noise had to be removed before further processing. Noise segmentation was done by grouping points using Hausdorff distance. Removal of redundant, protruding point groups spread across the measurement volume was then performed to ensure the consistency of the scan. Additionally, boundary of each cloud was removed because of the highest inaccuracy errors accumulating there. The 8-directional point clouds were initially aligned and overlapping, but fine alignment was still required. The Iterative Closest Point (ICP) method [7] was chosen to refine the relative positions of the clouds. Finally, they could be merged into one, complete 3D whole body scan.

When the 3D scan of the subject was ready, the actual anthropometric measurement extraction process began. It started with the preprocessing stage including dataset segmentation into typical body parts, i.e. arms, legs, upper/lower torso, hips, and head [8]. The exact feature extraction was preceded by a fully automatic body landmarking. The garment design and characteristic points had to be localized prior final measurements, as they determined the measurement path. Calculations of Gaussian curvature and analysis of shape features enabled localization of landmarks [8].

As each measurement was different, the approach on how to calculate them differed as well. Three main approaches could be distinguished: girths, arc lengths, and linear sizes including widths and heights. The way the measurements were grouped is shown in figure 2. Most of the measurement algorithms worked on a specific volume of interest (VOI), based on the previously segmented body parts, to which the sought dimension belonged (e.g. hip, leg, calf etc.). The plane in which lied the measurement had to be defined and localized using specific features. For example, the chest girth was defined as parallel to the ground level and crossing the nipples. On the other hand, arc lengths measurements were constrained by the specific points like acromions, cervical, or elbows. When the measurement plane was found, a slice of corresponding point cloud was extracted with a certain thickness equivalent to the measurement tape width. In the girth case, the segmented points were projected onto the measurement plane and the $2 \mathrm{D}$ convex hull was computed, which resembled the way a measurement tape works. Arc lengths computation was similar, however additional convex hull clipping, based on a garment characteristic points, had to be performed. Linear measurements were more straightforward as they were calculated as Euclidean distances between specific body landmarks or other auxiliary points. Straddled posture was considered and compensated while measuring heights by virtual repositioning of the leg positions to imitate the subject's upright posture.

More detailed description of the used algorithms and their implementations can be found in [8].

\subsection{Algorithms validation}

The quality of the measurement methods had to be verified. During the preliminary test phase, a group of 40 volunteers ( 21 male and 19 female) was measured manually by a trained operator and automatically by both OGX|MMS and Human Solutions systems. In this case, manual anthropometric measurements were treated as a gold standard. 26 measurements common for both systems were chosen for the analysis. In order to assess the reliability of the systems, the relative percent and 
millimeter difference errors were calculated. Consistency and absolute agreement between automated and manual measurements were evaluated using the Intraclass Correlation Coefficient (ICC) method [9]. Validity of manual and automated measurements comparison was assessed according to ISO 20685 [8].

\subsection{Practical system validation}

During the practical system validation phase, 32 police officers were scanned. In this phase manual measurements were not performed and the test results relied purely on the OGX|MMS system. Tailored clothing is currently being prepared for each of the persons and fitting quality will be evaluated.

\subsection{Updated size charts designation}

When the validation stage was finished, the actual population-wide survey could be conducted. Altogether 3364 officers (2802 male and 562 female) from four different formations were fully scanned. This included 1858 officers from the Police, 638 from the Border Guard, 233 from the Government Protection Bureau, and 635 from the Fire Service. 530 scans were performed by the newly developed OGX|MMS device and the rest by the Human Solutions system as the reference one. The output from both scanners was uploaded to a remote database, statistically analyzed and compared with the old size charts. In order to fulfill this stage, Medcore prepared an appropriate independent solution. It included a special 3D measurement loading module which established a bridge between the two scanning solutions as well as the analytic and evidence system (see figure 5). Both modules were connected to the remote database.

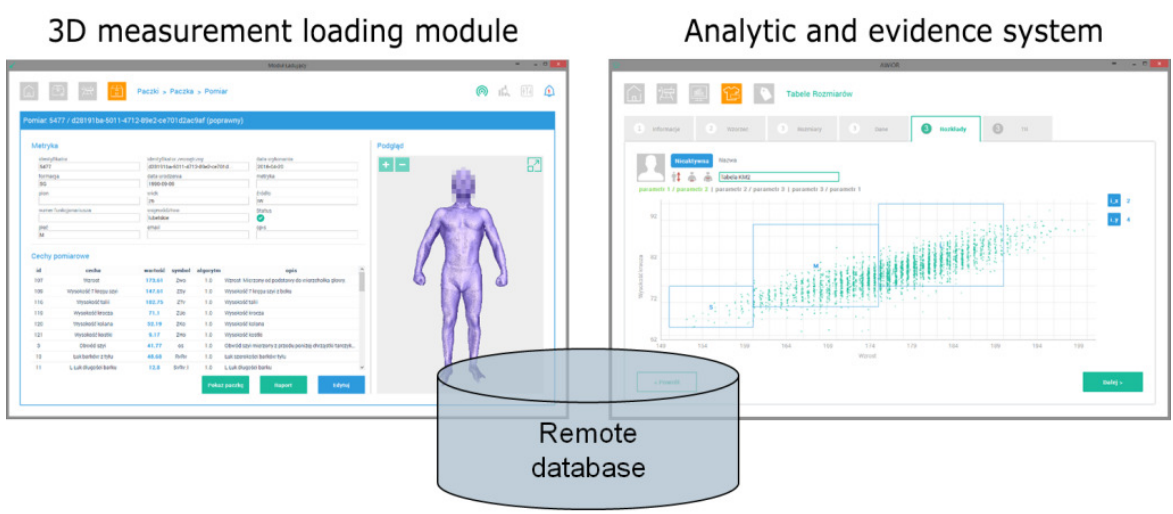

Fig. 5. Concept of the analytic and evidence system linked with scanning systems via 3D measurement loading module.

The last stage of the process, i.e. designation of the new size charts, is still ongoing and its results are not available yet. However, during this stage, the size charts are expected to be updated and optimized in a way to ensure the maximum possible coverage of the population. This way the number of badly fit uniforms should be significantly reduced.

In order to keep the sizing charts updated, as human body changes over time and the officer population varies, the last stage can be repeated periodically with the use of the proposed ready-to-use 3D anthropometric solution.

\section{Results}

The first tangible effect of this project was the fully functional and mobile OGX|MMS system equipped with a 3D whole body scanner and measurement software. Selected measurements obtained using OGX|MMS system are shown in figure 6. 


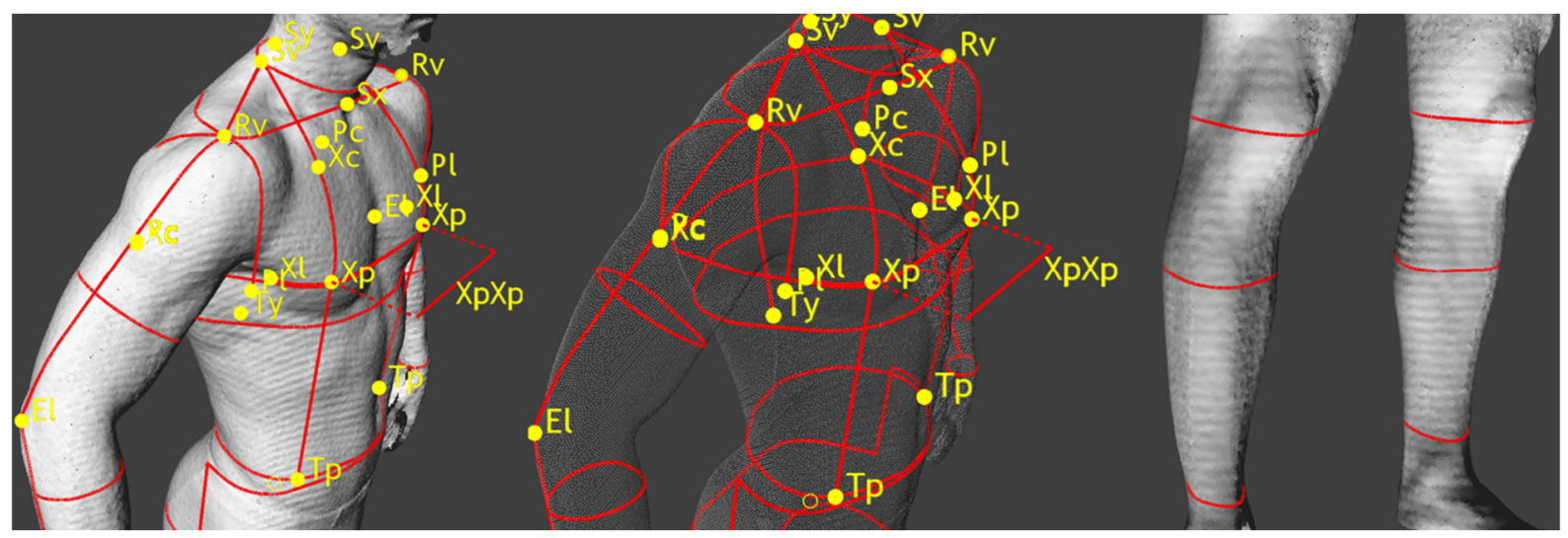

Fig. 6. Visualization of the selected measurement lines and body landmarks obtained by the developed OGXIMMS system.

The results of the measurement algorithms quality tests proved that the OGX|MMS system can provide results similar to those of the state-of-the-art solution used in the project. High inter-rater agreement between the OGX|MMS system and the commercial Human Solutions one was observed. According to garment design experts engaged in the project, most of the accuracy, consistency, and absolute agreement results appeared to be at a satisfactory level. The highest degree of consistency and absolute agreement was obtained in girth measurements. Arc length measurements were the least satisfactory, but at a still acceptable level. General consistency and absolute agreement degrees between the developed system and manual measurements fell into the excellent interval of the ICC practical significance measure. However, neither OGX|MMS nor Human Solutions systems passed the highly demanding test introduced in the ISO 20685 norm, which assesses the comparability between the manual and automatic measurements. It means that consistency between the traditional and automated 3D approach is not always preserved. These errors were mainly caused by the differences between postures during manual measurement and 3D scanning as well as, in a few cases, too fuzzy definition of body landmark localization due to its impalpable nature. These differences will have to be taken into account during future garment sizing chart designation in order to minimize the ambiguity and errors. A more detailed description of this validation process and its results can be found in [8].

In the next, practical, real-life system validation phase, a group of police officers was scanned. The tailor-made uniforms are currently knitted based only on data derived from OGX|MMS system and without any additional manual check. This ongoing test is expected to confirm the general OGX|MMS system output quality.

After successful preliminary system tests, the proper large-scale scanning survey began. The data was collected using both systems and is currently stored in the remote database. The software handling the data and responsible for statistical analysis is presented in figures 7 and 8.

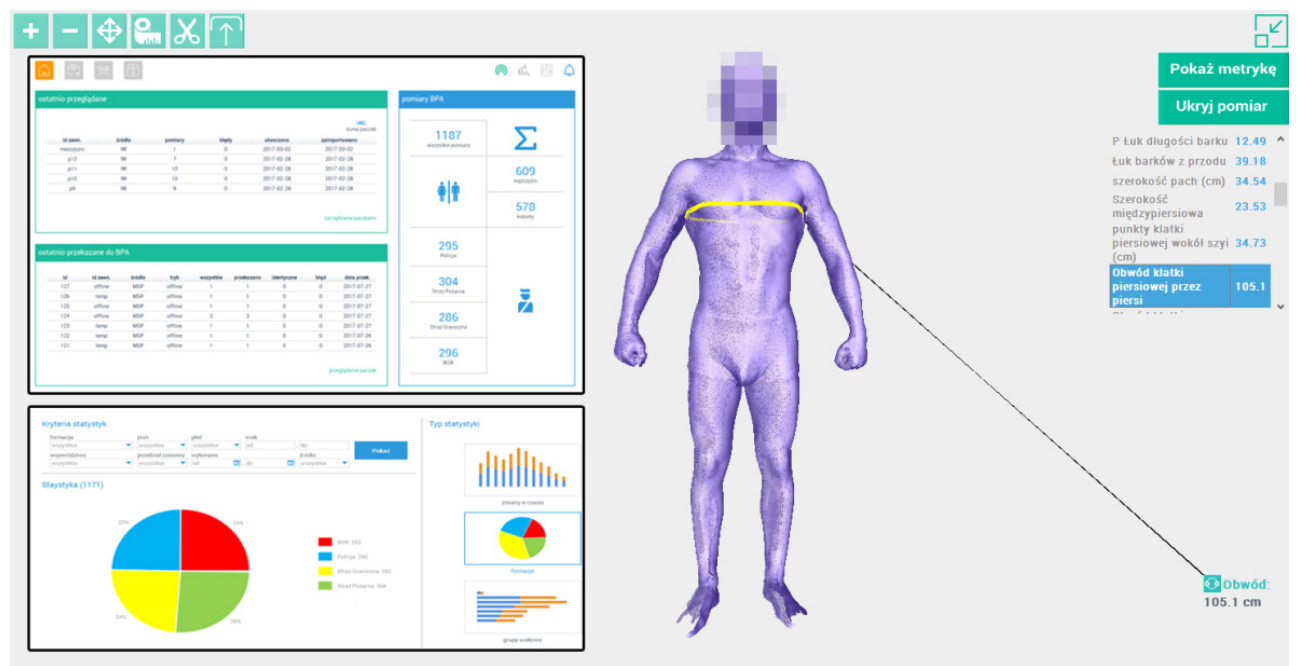

Fig. 7. The example views of the software handling the data gathered during the research. 
The final phase of the project is not finished yet and the new size charts are still under development. Nevertheless, the preliminary tests on the old size charts coverage were done. One of the example cases is presented in figure 8. It appeared that for some of the common garment types, e.g. winter police trousers, almost half of the measurements do not fall into any of the size tables intervals. This confirmed the legitimacy of the project, revealed the scale of the problem and ensured that the new updated size charts are necessary in order to prevent further magazine losses. Although it will still not be possible to provide a perfectly fitting uniform for every person, the general comfort of wearing the garment can be greatly improved.

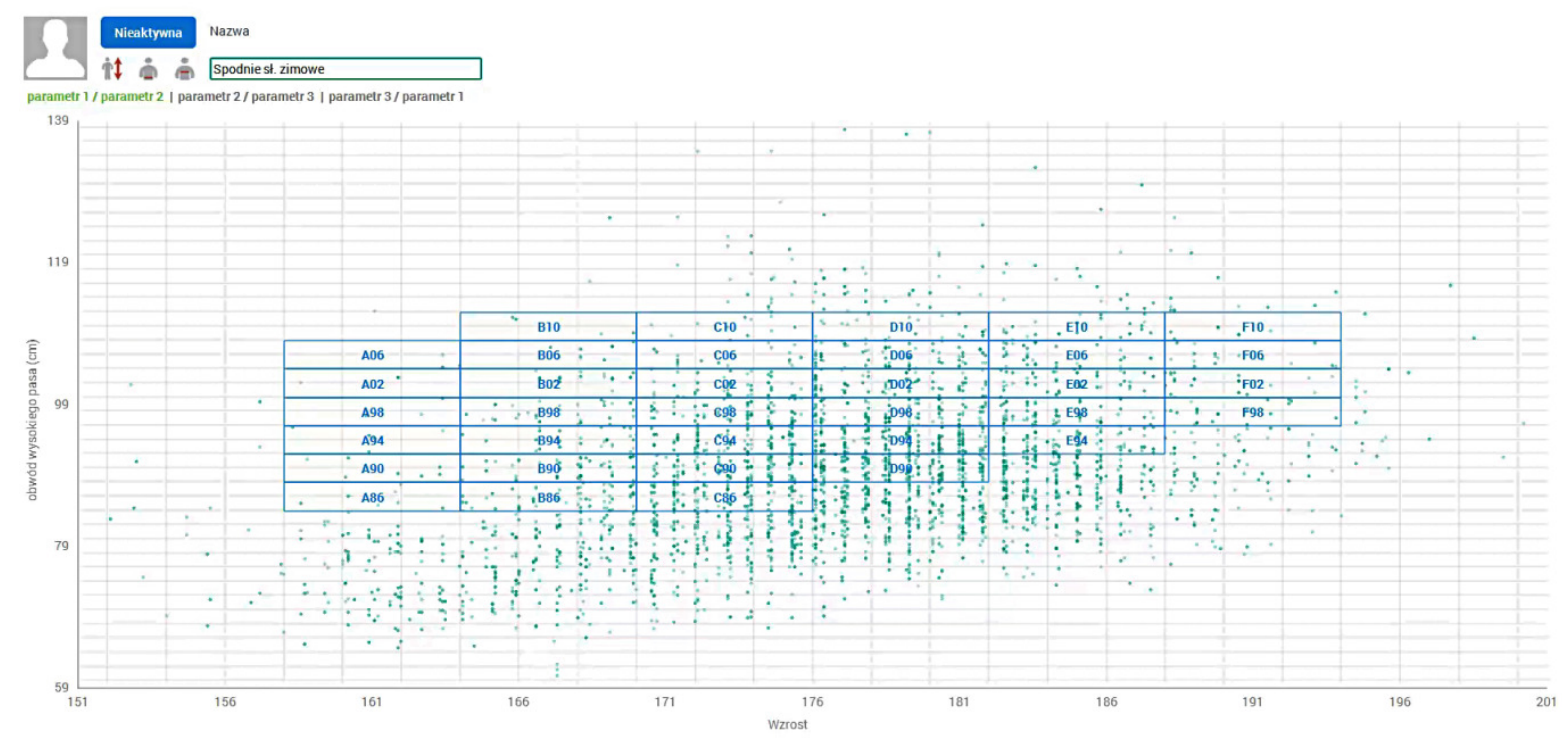

Fig. 8. The size charts analysis and optimization tool view, where $X$ axis is the height of a person, $Y$ axis his/her waist girth. Old size charts are represented by blue rectangles, and green dots correspond to measured persons (police winter trousers case).

\section{Conclusions}

In this study, the unification process for uniforms with use of 3D scanning was described and general guidelines for future large-scale surveys were presented. During the research a new comprehensive 3D anthropometric system was built. It consisted of the 3D mobile whole body scanner and the appropriate measurement software. It was linked with the remote database via the loading module operating on both OGX|MMS and Vitronic VITUS smart XXL scanners outputs. Moreover, the additional statistical analysis program was developed to enhance the designation process of the new size charts. Brief presentation of the research objectives met in this project is shown in figure 9.

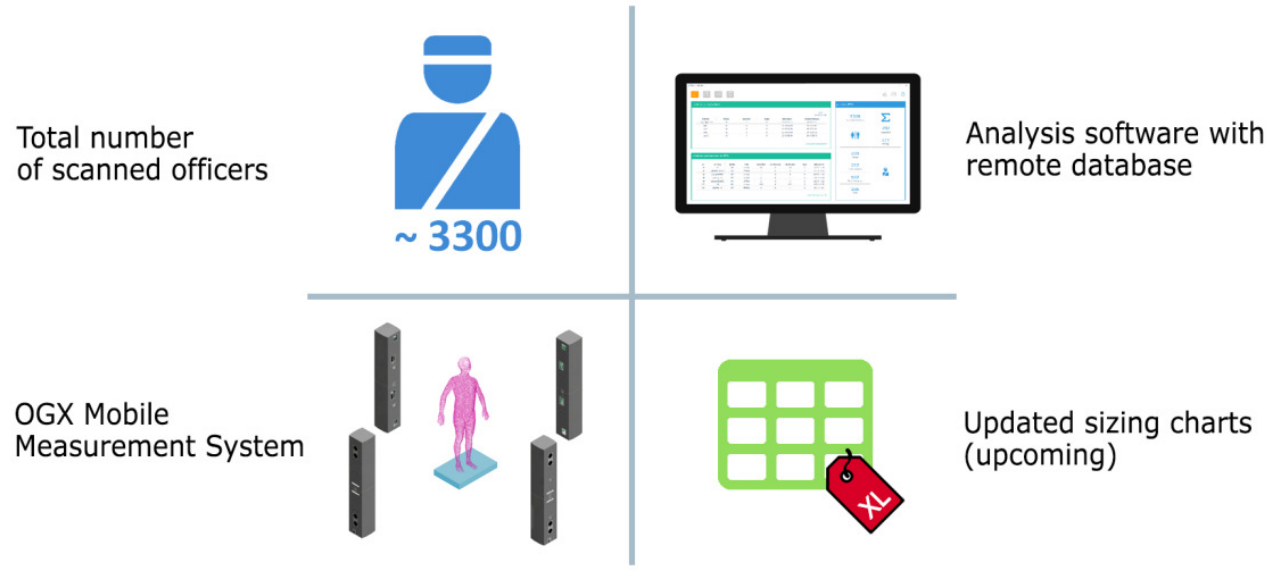

Fig. 9. Overview of the main research objectives met. 
The quality of the system was assessed in a series of test scans to ensure the reliability of the proposed 3D anthropometric solution. First set of the individually customized garment is currently tailored for the officers in order to validate the assumed methodology. Despite that until now the scanned persons were treated individually and new sizing charts are not ready yet, preliminary results were obtained from the whole scanned population data. They confirmed legitimacy of designation of new size charts. It appears that in some cases inconsistency between the real officers dimensions and the size charts can be up to $50 \%$, which means that almost half of the officers are not able to receive the properly fitting clothing of a certain type. Of course, some of the incorrectly fitted uniforms are used despite that, but this leads to frustration of the owner and is connected with a costly process of individual tailor amendments. Nevertheless, such inconsistency leads to huge money losses each year, when the unused and expensive specialized garments fill up and perish in the magazines. Additional advantage of the proposed $3 \mathrm{D}$ anthropometric solution is that measurements for the stored virtual models can be repeated, modified and extended in the future if needed. They can also be used as a reference in a future periodical monitoring which is possible thanks to the available OGX|MMS system. This way the cumulative cost of the iterative processes of the uniform fitting and evaluation can be reduced. Moreover, the proposed unification processing path is not constrained only to the Formfit project case and can be applied in other fields. Overall, it is expected that the developed system will help to significantly improve the garment fitting quality for the officers, reduce the magazine losses and ensure consistency across the uniforms size charts between different Polish uniformed services.

\section{Acknowledgment}

This work has been financially supported by the National Centre for Research and Development as part of the project DOB-BIO6/20/74/2014, realized within the Defence and Security Programme (2014-2017) and statutory work of IMiF Warsaw University of Technology.

\section{References}

[1] R. Sitnik, M. Kujawińska, and J. Woźnicki, "Digital fringe projection system for large-volume 360-deg shape measurement", in Opt. Eng., vol. 41, no. 2, 2002, pp. 443-449, http://dx.doi.org/10.1117/1.1430422.

[2] R. Sitnik, "New method of structure light measurement system calibration based on adaptive and effective evaluation of 3D-phase distribution", in Proc. SPIE, vol. 5856, 2005, pp. 109-117, http://dx.doi.org/10.1117/12.613017.

[3] Instytut Technik i Technologii Dziewiarskich TRICOTEXTIL, Tabele szczegółowych wymiarów dzieci, dziewcząt, chłopców, kobiet i mężczyzn. Łódź, 1997.

[4] H. a. M. Daanen and F. B. Ter Haar, "3D whole body scanners revisited", in Displays, vol. 34, no. 4, Oct. 2013, pp. 270-275 http://doi.org/10.1016/j.displa.2013.08.011.

[5] "Human Solutions web page". [Online]. Available: http://www.human-solutions.com/. [Accessed: 31-Aug-2017].

[6] "Vitronic advertising materials", $2012 . \quad$ [Online]. Available: https://www.vitronic.com/industrial-and-logistics-automation/sectors/3d-body-scanner/3d-scan-forcustomized-clothing.html. [Accessed: 31-Aug-2017].

[7] P. J. Besl and N. D. McKay, "A Method for Registration of 3-D Shapes", in IEEE Trans. Pattern Anal. Mach. Intell., vol. 14, no. 2, 1992, pp. 239-256, http://dx.doi.org/10.1109/34.121791.

[8] Ł. Markiewicz, M. Witkowski, R. Sitnik, and E. Mielicka, "3D anthropometric algorithms for the estimation of measurements required for specialized garment design", in Expert Syst. Appl., vol. 85, 2017, pp. 366-385, http://dx.doi.org/10.1016/j.eswa.2017.04.052.

[9] P. E. Shrout and J. L. Fleiss, "Intraclass correlations: uses in assessing rater reliability", in Psychol. Bull., vol. 86, 1979, pp. 420-428, http://dx.doi.org/10.1037/0033-2909.86.2.420. 